\title{
Adaptación de la asignatura de Responsabilidad Social del grado de Periodismo a la docencia en línea, en tiempos de COVID-19
}

\section{Adapting a university subject to online learning in times of COVID-19: a chance to improve}

\author{
Carmen de la Calle Maldonado \\ Universidad Francisco de Vitoria. Madrid, España \\ m.calle@ufv.es \\ Susana Miró López \\ Universidad Francisco de Vitoria. Madrid, España \\ s.miro@ufv.es \\ Teresa de Dios Alija \\ Universidad Francisco de Vitoria. Madrid, España \\ t.dedios.prof@ufv.es \\ Daniel de la Rosa Ruiz \\ Universidad Francisco de Vitoria. Madrid, España \\ d.delarosa@ufv.es
}

\section{Resumen}

La COVID-19 ha sido una oportunidad de mejora en la docencia de la asignatura de Responsabilidad Social en la UFV. La urgente necesidad de que los medios de comunicación informen con veracidad ha adquirido mayor relevancia durante la pandemia y eso es algo que ha de aprenderse ya desde la Universidad. La formación en valores como la ética y la justicia social de los estudiantes de periodismo también puede hacerse a través del aprendizaje-servicio en línea.

Se procede a hacer un descriptivo de la asignatura, así como su adaptación curricular por el confinamiento utilizando nuevos recursos. Realizamos un análisis cualitativo, mediante Atlas.ti sobre el material producido por los estudiantes, para mostrar cómo estos se han convertido en agentes de cambio social.

La principal consecuencia es el aumento significativo del grado de responsabilidad social del universitario durante la pandemia mediante formas de comunicación diferentes a las habituales. La pandemia ha supuesto una oportunidad para replantear la asignatura y ha enriquecido la experiencia del universitario en la búsqueda de soluciones colaborativas y de ayuda, reforzando su compromiso social con el bien común a través de las nuevas tecnologías.

Palabras claves: COVID-19, comunicación, responsabilidad social, formación, acompañamiento.

\begin{abstract}
COVID-19 has been an opportunity to improve the teaching of Social Responsibility at the UFV. The pandemic has increased the urgency of trustworthy mass media and that is something to be taught at University. Training in values such as ethics and social justice for journalism students can also be done through online servicelearning.
\end{abstract}

RED. Revista de Educación a Distancia. Núm. 65, Vol. 21. Artíc. 12, 08-01-2021

DOI: http://dx.doi.org/10.6018/red.449841 
We proceed to make a description of the subject, as well as its curricular adaptation using new resources due to confinement. We carry out a qualitative analysis, applying Atlas.ti on the material produced by the students, to show how they have become agents of social change.

The main consequence is the significant increase in the degree of social responsibility of the university student during the pandemic through different forms of communication from the usual ones. The pandemic has provided an opportunity to rethink the subject and has enriched the experience of the university student in the search for collaborative solutions and help, reinforcing their social commitment to the common good through on line learning.

Keywords: COVID-19, communication, social responsibility, training, accompaniment.

\section{Introducción}

La COVID-19 ha irrumpido de forma estrepitosa en nuestras vidas, el 2020 pasará a la historia como el año de la pandemia y el confinamiento. Hoy más que nunca, ante esta inesperada y caótica situación, la creencia de que "quien tiene la información, tiene el poder" se ha presentado como una máxima irrebatible. La necesidad de saber, de conocer la verdad sobre lo que realmente está pasando, se ha convertido casi en una cuestión de supervivencia (Ballesteros, 2020). Al menos, así lo experimentan muchos de los que acuden constantemente a los medios de comunicación de masas y a las redes sociales para estar al tanto de lo que sucede en tiempo real (recomendaciones sanitarias, número de contagios, personas fallecidas, medidas del estado de alarma, restricciones, rebrotes...). Ante la incertidumbre y el miedo, las personas buscan certezas sobre la COVID-19 en los medios de comunicación para sentirse más seguros aunque, a veces, dada la disparidad de las noticias provoque más dudas (Catalán-Matamoros, 2020). Es preciso que "la información provenga de fuentes fiables y haya sido contrastada por los especialistas" (Cardoza, 2020, párr. 7). La información, en nuestra denominada "Era de la información", se ha convertido en un fin en sí misma, desde la firme convicción de que la información es deseable por sí y en el mayor volumen posible. Parece que lo importante es disponer del máximo número de datos, de cualquier manera y a cualquier precio: cuantos más, mejor (Terol-Bolinches \& Alonso-López, 2020).

Pasado el primer tsunami informativo y sabiendo que el coronavirus ha llegado para quedarse un tiempo impredecible entre nosotros, surgen ahora desde la Academia muchas preguntas (Adelson, 2020; Du Preez, 2020). Es momento para la reflexión, el balance y la toma de decisiones y medidas ante lo que nos depara un futuro incierto: ¿para qué tanta información?, ¿con qué propósito esta sobreabundancia?, ¿realmente estamos informados de lo que está aconteciendo?, ¿qué sentido tiene la saturación de datos?, ¿hay una censura intencionada en lo que nos llega?, ¿cómo garantizar que las noticias no sean falsas o manipuladas?, ¿cómo impacta todo esto en medio del caos sanitario, económico y social sufrido?

No es fácil responder a estos interrogantes sin adentrarnos en la cuestión del sentido, del para qué, y también del cómo y del qué. Es necesario pues hacer una inmersión en el ámbito de la ética y la deontología de los profesionales de la

Adaptación de una materia universitaria al aprendizaje en línea en tiempos de COVID19: una oportunidad de mejora. De la Calle, Miró, de Dios y de la Rosa. 
comunicación que tienen la responsabilidad social de informar con objetividad y verdad (Agejas \& De Dios, 2016). Este deseo de saberlo todo, propio de la naturaleza humana pero llevado en ocasiones al extremo en la Postmodernidad, tiene su origen en el ideal de dominio de la Ilustración. Se fundamenta en la creencia de que estar informado supone una cierta soberanía sobre la realidad que nos rodea. Es como si saberlo todo fuera una forma de poder y de control tanto de la propia vida como de las de los demás.

Sin embargo, la realidad siempre acaba imponiéndose: la especie humana es vulnerable por naturaleza y por mucho que persiga el mito del eterno progreso, sigue estando a merced de lo que venga y no es capaz de controlar (Bodenhausen \& Peery, 2009); en esta ocasión, estamos ante un virus aparentemente invisible pero letal, que provoca miles de muertos en un corto espacio de tiempo, tal y como ya ocurrió en el pasado con otras epidemias (Marcos, 2020). En medio de una sociedad avanzada, con unos niveles de progreso tecnológico y científico impensables en siglos anteriores, la humanidad del mundo globalizado del siglo XXI se siente amenazada por una nueva pandemia que lo infecta y afecta a todo, incluido el derecho a la información. Las fake news y los bulos que circulan libremente y a una velocidad trepidante como nunca antes, provocan, al mismo tiempo y de forma paradójica, una sensación de desinformación, en una población asustada, aislada, confinada y especialmente expuesta a una posible manipulación.

En abril de 2018 la Unión Europea difundió los resultados de una consulta pública sobre la circulación de fake news y de desinformación a través de medios online (Comisión Europea, 2018). En ella participaron 2.784 ciudadanos y 202 periodistas y organismos legales de países como Bélgica, Francia, Reino Unido, Italia, Lituania, Eslovaquia y Rumanía y España, entre otros. Los datos que expresa la encuesta son claros: el 37,6\% de las respuestas de ciudadanos preguntados indican que diariamente se encuentran con fake news o desinformación. Mientras que un 31,8\% manifiesta encontrar en sus redes fake news cada semana.

Esto significa que continuamente estamos expuestos a noticias falsas. Hay un denominador común a todas las fake news: una vez que una noticia falsa es difundida, los esfuerzos para combatirla resultan casi siempre inútiles o son tan complejos que ofrecen respuestas que contrastan los datos falsos de manera poco eficaz o llegan tarde, cuando ya se ha tomado como verdad entre millones de usuarios de redes sociales. Sin duda la inteligencia artificial y blockchain consiguen prevenir que aumente la velocidad de propagación, pero también surge con su uso un temor: ¿podrían ser programadas en contra de la libertad de expresión? Para prevenir y combatir estos riesgos los ciudadanos de todas las edades y profesiones deberían tener una idea clara de cómo trabajan los medios de comunicación, los periodistas, pero también conocer mejor las redes sociales como medio. La encuesta muestra cómo la mayoría de las organizaciones y de periodistas $(74 \%)$ creen que el público no está suficientemente preparado para chequear la veracidad de las noticias, para comparar y comprobar distintas fuentes, etc. Por otro lado, los ciudadanos (70\%) piensan que las plataformas de redes sociales no están haciendo lo suficiente para ayudar a sus usuarios a chequear los contenidos publicados antes de compartirlos. En lo que sí aparecen de acuerdo organizaciones y periodistas es en que todavía se puede hacer mucho más para evitar la difusión de desinformación (Centro de documentación europea UFV, 2020).

Adaptación de una materia universitaria al aprendizaje en línea en tiempos de COVID19: una oportunidad de mejora. De la Calle, Miró, de Dios y de la Rosa. 
En este contexto parece esencial articular medidas y protocolos necesarios para que las noticias que acaparan los medios de comunicación y las redes sociales no escapen al control deontológico (Morelo et al. 2020). Esto ha de ser así siempre, pero muy especialmente en momentos críticos como éste. Las consecuencias de la desinformación, el exceso de información o la información manipulada pueden agravar más si cabe las nefastas consecuencias de la pandemia. La actualidad y el futuro inmediato y mediato demandan comunicadores socialmente responsables, que informen con veracidad, sin manipular, con un código deontológico y una ética profesional a prueba de virus y crisis del tipo que sean. La misión que asume la Universidad al ofrecer una formación verdaderamente integral a los estudiantes supone responder a la necesidad de que los futuros profesionales desarrollen su trabajo desde una posición de compromiso social (De la Calle \& Giménez-Armentia, 2011). Es urgente educar en responsabilidad a los jóvenes y no nos podemos permitir perder una generación por falta de medios o dificultades añadidas por la COVID-19. La emergencia no es solo sanitaria, también es educativa (CEPAL, 2020).

En el contexto universitario se está trabajando en la adquisición de competencias y cualificaciones que mejoren la empleabilidad de los jóvenes, así como su participación en la sociedad (Hernández de Hahn, 2014). La capacidad de pensar críticamente y hacer juicios equilibrados sobre cualquier información se ha convertido en una competencia clave para los universitarios, en especial para los que hoy estudian Ciencias de la Información (Landøy, Popa \& Repanovici, 2020). Es misión de la Universidad, especialmente de las Facultades de Comunicación, pero también en otras faultades que se dedican a otros saberes dado que el juicio crítico es competencia indispensable para todo profesional que se precie, permitir a los estudiantes adquirir una dimensión ética en su formación para que se conviertan en profesionales socialmente responsables en toda su trayectoria, pero de manera más patente en situaciones como las que estamos viviendo.

La Universidad no puede eludir el compromiso social que supone educar a los jóvenes para ser capaces de dar respuestas a los retos futuros, unos retos que, como con la COVID-19, resultan insospechados. La formación en responsabilidad social es vital para poder enfrentarse a situaciones tan críticas como las que estamos padeciendo. Por ello, en todos los grados universitarios que se cursan en la Universidad Francisco de Vitoria (en adelante UFV) se trabaja en el desarrollo de competencias que estimulan el compromiso y la responsabilidad social, facilitando la reflexión acerca de las implicaciones de la naturaleza social en la convivencia humana, permitiendo conocer la situación del mundo hoy, tanto próximo como remoto y dando sentido al desarrollo pleno e integral de la persona mediante la comprensión de factores clave como la dignidad humana y la solidaridad. En concreto en el Grado de Periodismo, enmarcado en la Facultad de Ciencias de la Comunicación, esta formación se articula a través de las materias de Antropología, Responsabilidad Social, Ética y Deontología profesional. La sociedad necesita cada vez y con mayor urgencia profesionales del periodismo socialmente responsables y muchos de ellos se están formando ahora en la universidad, de ahí la emergencia educativa que se precisa (Reason et al. 2013; Larrán, 2014; De Dios, 2018).

Adaptación de una materia universitaria al aprendizaje en línea en tiempos de COVID19: una oportunidad de mejora. De la Calle, Miró, de Dios y de la Rosa. 
En este trabajo presentamos cómo se ha adaptado el programa formativo que incide en el compromiso social, a raíz de la situación generada por la COVID-19 y cuáles han sido sus efectos en el alumnado y en la comunidad universitaria. Se nos planteaba la necesidad de mantener el objetivo formativo en un marco educativo totalmente inesperado respecto al momento en que se habían elaborado las guías docentes. Entendimos que era preciso un cambio metodológico para alcanzar la finalidad educativa. Presentamos la adaptación realizada y cómo se ha enriquecido la experiencia del universitario, reforzando su compromiso con el bien común a través de las nuevas tecnologías. Pese a los augurios más pesimistas, podemos afirmar que la COVID-19 se ha convertido en una oportunidad de mejora en la Universidad, aunando esfuerzos, proponiendo soluciones tecnológicamente más creativas y provocando en el alumno un sentido de mayor responsabilidad en su propia tarea formativa.

\section{Marco teórico: Educación para la Responsabilidad Social en la Facultad de Comunicación}

La responsabilidad social es un valor en auge en la actualidad en todos los campos profesionales. En este momento de crisis sanitaria y económica globalizada interesa especialmente reconocer su incidencia en la labor de los medios de comunicación, cuya credibilidad está en juego más que nunca. Es clave pues, garantizar que lo que comunican está contrastado con fuentes rigurosas y fidedignas y que cumplan de forma ética con su labor informativa (Agejas \& De Dios, 2016). El criterio para discernir cuándo se informa con responsabilidad ha de formarse también en el ámbito educativo a lo largo de sus distintas etapas, y especialmente en las universidades.

La UFV tiene como materia de obligado cumplimiento la asignatura de Responsabilidad Social para que los universitarios descubran su compromiso con el bien común y con la búsqueda de la verdad y su responsabilidad en la construcción de un mundo más justo y solidario, hoy como universitarios y el día de mañana como profesionales. Esta materia está integrada por una parte teórica en el aula, unas prácticas de participación en distintos proyectos sociales y un programa de acompañamiento (De la Calle \& Giménez-Armentia, 2011; García Ramos et al. 2016). Se ha impulsado la puesta en marcha de esta asignatura con la convicción de que la formación universitaria ha de abordarse desde la perspectiva del desarrollo integral de la persona, superando una concepción que atienda exclusivamente a la preparación técnica de los futuros profesionales (Ortiz \& Sanz, 2016).

El curriculum que desarrollan los planes de estudios de esta universidad plantea en todas sus titulaciones una serie de créditos de materias humanísticas con el objetivo de desarrollar valores, actitudes y hábitos que propicien en los alumnos el pensamiento crítico en el análisis de la realidad y un ejercicio responsable desde un prisma ético en el desarrollo de su conciencia personal como agentes sociales y en la toma de decisiones de su futuro profesional.

En este contexto de materias de corte humanístico se encuentra la asignatura de Responsabilidad Social que hace suyo el lema de la UFV "Vince in bonum malum" e invita a los jóvenes a "atreverse a cambiar el mundo", despertando su interés por la

Adaptación de una materia universitaria al aprendizaje en línea en tiempos de COVID19: una oportunidad de mejora. De la Calle, Miró, de Dios y de la Rosa. 
atención a las personas con las que se relacionan en su día a día y saliendo también al encuentro de la realidad de las personas en entornos o situaciones de precariedad o vulnerabilidad, con intención de adoptar actitudes y compromisos sociales que puedan asumir como reto a lo largo de toda su vida personal y profesional.

En concreto, en la formación integral de los estudiantes que serán los futuros profesionales de los medios de comunicación adquiere pleno sentido la obligatoriedad de una asignatura como Responsabilidad Social, que ha sido tratada con ese peso desde el inicio de la UFV en 1993. Desde la actividad periodística y la influencia de los distintos medios de comunicación y las redes sociales se asume un liderazgo de suma importancia que puede ejercerse en el servicio a la sociedad y en la búsqueda de la verdad o decantarse a favor de intereses de tipo económico o ideológico más o menos direccionados.

Los profesionales de la comunicación han de ser sensibles a los contrastes escandalosos que se dan en el mundo globalizado y no ceder ante las presiones para descartar e ignorar la vulnerabilidad humana y la injusticia social. Su deontología profesional se pondrá de manifiesto aportando desde sus puestos de trabajo informaciones veraces que huyan del sentimentalismo, el sensacionalismo o la manipulación. El objetivo es que promuevan compromisos estables en el tiempo y propuestas concretas para trabajar con un rigor periodístico que posibilite la mejora de la sociedad (Agejas \& De Dios, 2016).

En este marco teórico que sostiene nuestro estudio, empezamos explicando por qué es coherente incluir la formación en la responsabilidad social en el proyecto educativo de la UFV. Luego presentamos los objetivos de la asignatura y su estructura. A continuación, describimos cada uno de los ámbitos en los que se desarrolla la materia de Responsabilidad Social entendida como una unidad: en el aula, en las prácticas sociales y en el acompañamiento personal. Y terminamos presentando la adaptación que hemos tenido que llevar a cabo durante el confinamiento por la pandemia que, lejos de suponer un menoscabo en los resultados de aprendizaje que buscamos, ha abierto nuevas posibilidades de formación en la ética profesional de los que trabajan en los medios de comunicación.

\section{Justificación de la formación en deontología profesional en la universidad}

Una asignatura como esta, que tiene como objetivo formar en la responsabilidad social, dada su novedad, exige por nuestra parte una breve explicación que permita afianzar aún con más claridad tanto la exigencia académica como el alcance formativo de la misma.

Es cierto que una asignatura de este tipo corre el peligro de algunas formas de educación moral contemporánea, que se reduzca a "una llamada que estimula ante todo nuestra sensibilidad y arraiga, en la mayor parte de los casos, sólo en el corazón entendido un sentido reduccionista-" (Álvarez, 2007, p.14). "Los niveles de solidaridad que se dan entre la población española son de los más altos de Europa, pero estos datos positivos, tienen la réplica de quien ve en la solidaridad española una solidaridad de momentos (pasión latina), lo que sólo palia problemas de corto plazo y no transforma problemas de raíz" (Osorio, 2003, p.55).

Adaptación de una materia universitaria al aprendizaje en línea en tiempos de COVID19: una oportunidad de mejora. De la Calle, Miró, de Dios y de la Rosa. 
Es esencial responder a esta cuestión: ¿cuáles son las verdaderas razones para asumir en primera persona un compromiso social que no se base tan sólo en "buenos sentimientos"? ¿Qué hace posible que nada que de lo humano pueda serle al ser humano ajeno o indiferente, especialmente el sufrimiento? La responsabilidad social responde a estas cuestiones sin servir a las modas y sin guiarse sólo de impulsos a merced de un emotivismo sin compromiso. La responsabilidad social es la que se fundamenta tanto teórica como prácticamente en el reconocimiento y verificación de la dignidad humana personal. Esto es, en el estudio, encuentro, contraste y vivencia en primera persona de las exigencias concretas que me plantea como sujeto y como profesional la relación con la vulnerabilidad de los otros y de la sociedad en la que vivo y a la que me preparo a servir (De la Calle \& Giménez-Armentia, 2011). Sólo si sabemos quién es el ser humano y le damos su valor más profundo sin depender de su situación y experiencia, ni de factores como la edad, el sexo, las condiciones físicas, intelectuales, psíquicas o culturales llegaremos a formar en verdadera responsabilidad social. De esa valoración emana directamente la exigencia universal de un trato conforme a las necesidades de la dignidad humana y la responsabilidad social de cada ser humano por atender a estas necesidades (De Dios, 2018).

Formar en la responsabilidad social consiste en dirigirse "en primer lugar hacia un descubrimiento gradual del otro y en segundo lugar hacia la participación en proyectos comunes." (Ortiz de Montellano, 2000, p.10). "La responsabilidad, que viene del latín, re: voltear y espond: promesa, se concibe entonces como la capacidad de voltearse, notar, verse afectado, informarse, crear vínculos y prometer" (Ortiz de Montellano, 2000, p.99).

López Quintás señala que en su etimología la palabra responsabilidad comparte raíces con los términos corresponder y corresponsable. De ahí se desprende el significado por el que "ser responsable" es responder o atender a la llamada de los valores que esperan cumplirse. El desarrollo personal de cada ser humano depende del descubrimiento de sus propios valores y del paso de su ser en potencia a ser en acción. El compromiso por que los valores se cumplan despliega en la realidad ciertas alternativas y selecciona frente a otros modos de ser y actuar una forma que responde a nuestra conciencia humana. Aunque en apariencia se limitan las posibilidades, en realidad se cumple la naturaleza del ser en forma de ob-ligación como "aquello a que lo que se está ligado", que nos corresponde. De ahí se deriva la definición de auténtica libertad que sirve de fundamento a la obligatoriedad de la asignatura de Responsabilidad social: la libertad como vinculación ligada a los valores. El diseño de esta materia universitaria incluye como elemento esencial la participación en las prácticas de acción social. Se hace necesario insistir sobre qué entendemos por obligación el sentido en el que lo explica López Quintás como vinculación a algo valioso, sentirse ligado a algo que te realiza como persona (2003).

Estamos viviendo una situación en la que esto es clave para todos, y en especial para los jóvenes, que cuestionan las normas que les vienen impuestas desde fuera por la autoridad competente. Han de ser ellos mismos quienes caigan en la cuenta de la trascendencia que tienen sus actos individuales, que sean conscientes de que lo que hacen y cómo lo hacen, impacta en los demás y a veces, puede tener consecuencias imprevisibles y desastrosas para otros, especialmente para los más vulnerables.

Adaptación de una materia universitaria al aprendizaje en línea en tiempos de COVID19: una oportunidad de mejora. De la Calle, Miró, de Dios y de la Rosa. 
Puede resultar difícil o paradójico enseñar un valor que se ha de asumir libremente, voluntariamente, en nuestro caso el de la responsabilidad social en una asignatura obligatoria. Lo que pretendemos es apoyar al universitario para que llegue a reconocerlo por sí mismo, es decir, que descubra lo valioso del encuentro con el otro y, por propia experiencia, se convencerá internamente de lo valioso que es la persona con independencia de las circunstancias que rodeen su vida. Esta asunción le marcará el resto de su trayectoria universitaria y profesional pero, solo si lo ha interiorizado como algo verdaderamente valioso (López Quintás, 2003).

A continuación, presentamos la estructura de la asignatura de Educación para la Responsabilidad Social. Esta materia está concebida como un todo, una unidad integrada por tres ámbitos que se interrelacionan, retroalimentan y se necesitan mutuamente: el aula, las prácticas sociales y el acompañamiento personal.

Las clases teóricas en el aula, tienen como objetivo provocar en el alumno una reflexión profunda sobre el fundamento de la responsabilidad social desde el respeto a la dignidad humana. Partimos de la posibilidad de cambiar nuestro entorno personal y profesional, asumiendo la parte de responsabilidad personal que cada uno tiene en la construcción de un mundo mejor, desde la inquietud de cambiar las situaciones de injusticia y desigualdad que vemos a nuestro alrededor.

En las prácticas sociales, el objetivo es hacer la experiencia en primera persona de que sí es posible hacer algo concreto para contribuir al cambio de muchos que están en una situación injusta, de marginación, soledad, sufrimiento, abandono, enfermedad... dando un poco de nuestro tiempo y de nuestra persona. Se pretende que el estudiante se acerque a otras realidades diferentes a las que él vive desde ese espíritu de apertura a lo universal propio del hecho de ser universitario. Abrirse al otro para encontrarse y para implicarse desde el compromiso personal. Para ello, el Departamento de Acción Social, encargado de coordinar y gestionar las prácticas sociales de los alumnos, asigna a cada alumno un proyecto social en el que participa a lo largo de todo el curso en colaboración con una de las 150 ONGs con las que trabajamos y que abarcan todos los campos de la acción social: discapacidad, adicciones, tercera edad, inmigración, reclusos, violencia contra la mujer y la infancia, jóvenes en riesgo de exclusión social, personas sin hogar....

El acompañamiento personal, busca facilitar al alumno su autoconocimiento y detectar sus necesidades, motivarle y lanzarle a la acción, provocar en él una reflexión profunda sobre el para qué de lo que está haciendo en relación con su vida personal y su futuro profesional. Acompañarle en ese descubrimiento personal que le permita conectar la experiencia vivida en la asignatura con su aquí y ahora, de cara a conseguir un aprendizaje significativo que contribuya a su formación integral.

Somos conscientes de que el valor de la responsabilidad social no se impone de forma coactiva, desde fuera, como algo extraño y ajeno; más bien se trata de mostrar que no podemos permanecer indiferentes a nada de lo que sucede a nuestros semejantes y en nuestro entorno; si queremos desarrollarnos plenamente como personas, estamos llamados al encuentro comprometido con los demás y a la búsqueda del bien común. Muchos de nuestros estudiantes han hecho este descubrimiento personal, esencial para su vida universitaria hoy y para el ejercicio responsable de su profesión en los medios de

Adaptación de una materia universitaria al aprendizaje en línea en tiempos de COVID19: una oportunidad de mejora. De la Calle, Miró, de Dios y de la Rosa. 
comunicación en el futuro, a través de esta asignatura. Y, una pandemia como la que estamos viviendo, ha puesto de manifiesto la necesidad de educar a los jóvenes de tal manera que estén dispuestos a asumir el reto de involucrarse en la sociedad porque nada de lo humano les puede ser ajeno y menos cuando un virus ha demostrado la vulnerabilidad de todo el orden mundial.

\section{Adaptación de la asignatura de Educación para la Responsabilidad Social en la Facultad de Comunicación por la pandemia de la COVID-19}

Como hemos recogido en el epígrafe anterior, para lograr los objetivos claves de la asignatura de Educación para la Responsabilidad Social articulamos la materia en tres partes: teoría, prácticas sociales y mentorías de acompañamiento. Los equipos docentes que conforman el claustro apoyan a lo largo del proceso formativo a los alumnos. Desde el departamento de Acción Social se genera una comunicación fluida con las instituciones donde los jóvenes realizan sus prácticas. Ante la COVID-19 ellos fueron los primeros en informar del posible riesgo de contagio en la nueva situación. A finales de febrero, distintas instituciones, sobre todo residencias de mayores y centros a atención a menores, se pusieron en contacto con la UFV para comunicarnos que, debido al nuevo virus que se estaba propagando por España no parecía conveniente exponer a las personas a un riesgo de contagio: ni a los alumnos que acudían a las organizaciones para realizar las prácticas sociales, ni a las personas que vivían en las diferentes instituciones con las que colabora la universidad. Los primeros en dar la voz de alarma fueron precisamente los centros de día, residencias de mayores y de personas con discapacidad.

Es lógico pensar que cualquier cambio genera inquietud en la comunidad universitaria, por ello desde el aula y desde el Departamento de Acción Social se informó a los estudiantes de que se establecería un plan de visitas para que pudieran continuar sus prácticas sociales cuando todo pasara. En esos momentos, las noticas que transmitían los diferentes medios de comunicación no parecían tan alarmantes, nos enfrentábamos a un nuevo coronavirus con unas tasas de contagio y mortalidad semejantes a las de la gripe, sin embargo, los responsables del Departamento de Acción Social reaccionaron con prontitud ante la más mínima posibilidad de poner en riesgo la salud de los beneficiarios de las prácticas sociales de nuestros estudiantes, así como la suya propia.

Al cabo de dos semanas, los medios de comunicación y las nuevas declaraciones de la Organización Mundial de la Salud (OMS) ofrecieron cifras sobre la magnitud del riesgo y todas las instituciones con las que tenemos acuerdos de colaboración de prácticas pidieron por cautela la suspensión de las mismas. El 10 de marzo también las clases presenciales se suspendieron de forma indefinida en el campus.

Las noticias, las cifras, los datos empezaban a ser alarmantes y desalentadores. Pensando sobre todo en el bien de los alumnos e intentando sumar desde la proactividad, tras varias reuniones de los distintos departamentos de la universidad y en medio de la alarma social, se buscaron alternativas para seguir con la docencia en remoto. Como profesores, nuestra prioridad era procurar que los alumnos completaran la formación correspondiente al curso académico 2019/20, también en responsabilidad social, porque estamos convencidos de que para transformar un mundo que ahora se ve envuelto en una crisis sanitaria, económica y social es necesario hacerlo desde la concienciación y la

Adaptación de una materia universitaria al aprendizaje en línea en tiempos de COVID19: una oportunidad de mejora. De la Calle, Miró, de Dios y de la Rosa. 
formación. La suspensión de la actividad docente hizo que se cambiase el método de aprendizaje y la metodología, siendo conscientes de que las medidas se han ajustado a la urgencia y es incomparable a propuestas que desde su inicio se concibieron para impartirse en formato online (Hodges et al. 2020), aun así, se puede considerar que la respuesta de la universidad fue aceptable, tal y como afirma en su estudio (GarcíaPeñalvoa et al. 2020). El miércoles 11 de marzo sustituimos el contacto de las clases presenciales por la docencia en remoto y se adaptó la asignatura de manera integral para que el aprendizaje de los estudiantes no se viese devaluado. Nuestra asignatura quedaba estructurada de la siguiente manera:

- Clases en remoto: seguimos manteniendo nuestros horarios habituales. Haciendo uso de la plataforma blackboard ultra cada profesor abría su aula y los alumnos se sumaban a ella. El mensaje del día siempre debía ser propositivo. A través del contacto visual, aunque, fuera por una pantalla, es posible transmitir calma, decisión y cercanía al alumno. Algunos de los estudiantes habían regresado a sus ciudades e incluso a sus países de origen. Los datos estadísticos han reflejado un aumento de participación en las aulas por encima de la actividad presencial. El 95 por ciento de nuestros alumnos en Latinoamérica han seguido las clases en tiempo real, sabiendo que la diferencia horaria media es de 6 horas y que algunas de las sesiones empiezan a las 8:00 hora española, el esfuerzo era digno de alabar. Cuando se les ha preguntado, en los cuestionarios de control que usamos, por qué lo hacen, la explicación más habitual ha sido que así se mantenían de verdad en contacto con lo que estaba pasando en su aula y que preferían la comunicación directa en este espacio que dejar un comentario en un foro. El contacto con los alumnos usando la plataforma digital posibilitó alcanzar los objetivos propuestos es en la guía docente. Se ha observado un incremento en la asistencia a las clases y un aumento de participación por parte de los alumnos utilizando los chats que proporciona la plataforma. Terminado el curso se pasa un cuestionario en el que los alumnos ofrecen feed-back tanto cuantitativo como cualitativo de las clases, nos ha sorprendido que la mayoría de ellos las clases les han proporcionado un argumentario crítico para entender qué estaba pasando en el mundo con esta pandemia y algunos de los estudiantes más retraídos y tímidos en el aula dicen sentirse más cómodos para participar en las clases a través de los vehículos implantados. A la hora de la evaluación, se siguieron algunas recomendaciones ofrecidas por la CRUE en su informe publicado este año sobre los distintos procedimientos de evaluación no presencial y cómo implementarlo en las universidades españolas. Y, a la par, se atendió a la necesidad de cada alumno y su circunstancia.

- El acompañamiento personal (mentoría): consta de dos sesiones individuales y dos grupales. En el momento de la interrupción de las clases algunos de nuestros alumnos todavía no habían realizado su segunda mentoría individual y en ninguna clase habíamos mantenido la sesión de cierre grupal. Ambos encuentros, por la experiencia acumulada, son cruciales para analizar la experiencia que han vivido en el curso y para que, de cara a entender el compromiso ético futuro, sepan establecer una proyección para el resto de su carrera y de su vida profesional. Los mentores de la facultad, sirviéndose de las más diversas plataformas (zoom, jitsi, whatsap, facetime...) mantuvieron los encuentros con cada uno de sus mentorandos. La última sesión grupal, tiene uno objetivo importantes en el desarrollo del aprendizaje de los alumnos, por un

Adaptación de una materia universitaria al aprendizaje en línea en tiempos de COVID19: una oportunidad de mejora. De la Calle, Miró, de Dios y de la Rosa. 
lado, invitar al alumno a profundizar en los encuentros mantenidos en las prácticas sociales para invitarles a continuar el camino y, por otro, hacer un ejercicio para transferir lo aprendido a su entorno más cercano (familia, clase, amistades, ...). Esta última sesión de acompañamiento requirió de unos soportes técnicos adecuados y de mucho esfuerzo personal para que el clima formativo fuera el idóneo. Se tuvieron formaciones específicas para que los mentores y profesores pudieran adaptar la sesión al formato on line. Las participaciones en estas sesiones fueron en torno al $95 \%$ en los diferentes Grados de la facultad de Comunicación y todos manifestaron un alto grado de satisfacción con esas sesiones grupales en los cuestionarios de evaluación posterior. Pese a que algunos de los estudiantes viven en lugares con más difícil acceso a internet, a través de sus dispositivos móviles pudieron participar sin ningún problema de estas sesiones. Valoran la flexibilidad y disponibilidad de las figuras de los mentores en el proceso. Hay que comentar que generacionalmente existe cierta brecha tecnológica entre los docentes y estudiantes, los propios alumnos se convirtieron en profesores de sus mentores para gestionar que la comunicación con las plataformas utilizadas fuera fluida, implicándose totalmente en el proceso de aprendizaje.

- Prácticas sociales: ha sido la parte más afectada de la asignatura, no ya porque fuera a resultar imposible la asistencia física a las instituciones, sino porque la mayoría de los alumnos, pasados ya seis meses de acudir semanalmente a los centros, habían generado un vínculo con las personas que allí se encuentran y no era fácil de explicar la razón por la que ya no iban a visitarles. Ante la dificultad había que generar nuevos escenarios de aprendizaje y por ello se propusieron la transformación de las prácticas sociales al uso en una serie de actividades sociales. Y entender por nuestra parte que la pandemia la debíamos poner en valor dado que ahora, durante el confinamiento, los estudiantes podrían hacer la experiencia en primera persona de la importancia de ser responsables como ciudadanos, vivirían lo que es el bien común no desde una definición teórica, sino desde la vivencia de cómo toda la comunidad está en riesgo (cuidándote a ti, cuidas de los demás; lo que uno hace nos afecta a todos; proteger a los más vulnerables es protegernos a todos...).

La pandemia ha puesto de manifiesto muchas de las debilidades de nuestros sistemas organizativos, pero también ha dejado rastro del buen hacer y de la generosidad de muchas personas. Nuestra responsabilidad era hacer llegar un mensaje a todas las instituciones sociales con las que colaboramos, de apoyo y acompañamiento, y en la medida de lo posible, que los alumnos de la universidad siguieran estando allí presentes. Igual que el equipo docente les acompañaba en esta nueva forma de enseñar en remoto, ellos debían ser agentes de esperanza en los centros donde estaban ayudando. A veces, no era posible o necesario en el lugar que tenían asignado, pero eran tantas las necesidades nuevas que surgían, que la colaboración de los alumnos ha sido de vital importancia (siempre extremando las medidas de seguridad y evitando riesgos).

Las prácticas sociales de los alumnos tuvieron que ser adaptadas al nuevo e inesperado contexto. En el caso de los estudiantes de periodismo, les propusimos una serie de actividades para llevar a cabo desde sus casas durante el confinamiento, en las que pudieran poner en juego su capacidad de servicio y desarrollar algunas competencias relacionadas con el arte de la comunicación.

Adaptación de una materia universitaria al aprendizaje en línea en tiempos de COVID19: una oportunidad de mejora. De la Calle, Miró, de Dios y de la Rosa. 
La manera de que ellos prestaran un servicio social en un momento de tanta demanda social y de necesidad de ayuda a todos los niveles, se articuló de la siguiente manera: partiendo del hecho indiscutible de que las personas mayores han sido las más afectadas por esta pandemia, y en especial, ellas son las que ha vivido con más intensidad el drama de la soledad y el aislamiento en las residencias de ancianos, pensamos que podía ser muy formativo y fecundo para nuestros futuros periodistas, encontrarse, a pesar de la distancia, con la realidad que se estaba viviendo en estos centros. La actualidad informativa pasaba por lo que estaba sucediendo en su interior, un espacio al que solo acuden los familiares o el personal sanitario y de gestión que trabaja en ellos. Sin embargo, nuestros estudiantes conocían esa realidad de primera mano porque durante los meses anteriores a la pandemia, muchos de ellos habían estado yendo una o dos veces por semana a acompañar a los ancianos que residen en ellas. Las noticias que ocupaban las portadas de los periódicos y abrían los telediarios hablaban de cifras y porcentajes a los que ellos podían poner un rostro humano concreto, con nombre y apellidos, personas a las que habían tenido la oportunidad de conocer en la distancia corta, en el tú a tú, en el encuentro personal. Y eso, lo cambia todo porque provoca que la noticia deje de ser algo aséptico y distante, para convertirse en una realidad cercana ante la que no se puede permanecer ajeno e indiferente porque "me afecta, me importa, me interpela...".

Además, algunos de nuestros estudiantes también han vivido en primera persona el horror y la angustia de perder a sus abuelos por esta enfermedad, sin poder despedirse de ellos o acompañarles en su final.

En este contexto difícil y duro, les propusimos una serie de actividades para seguir acompañando a nuestros mayores durante el confinamiento. Y qué mejor manera de hacerlo que invitándoles a poner en juego su creatividad y su capacidad de comunicarse a través de la palabra, instrumento clave en el ejercicio de su futura profesión como periodistas. Desde el Departamento de Acción Social se articularon los medios para llevar a cabo todo tipo de acciones con el objetivo de acompañar en la distancia: escribir cartas para hacérselas llegar a los que se encontraban más solos (mayores, enfermos, personas dependientes ....), dirigirlas a los que cuidaban a estas personas (no olvidemos que algunos sanitarios han pasado por unas situaciones muy duras y la recepción de una carta de agradecimiento les ha supuesto un punto de apoyo), grabar un vídeo y hacerlo llegar con un mensaje de ánimo a quien se le asignaba de forma personalizada, infografías para los más dependientes... los ejemplos son múltiples, parte de ellos recogidos en la propia cuenta de instagram del departamento: @ acciónsocialufv. De la misma manera que cada estudiante ha sido acompañado por sus profesores durante el confinamiento, cada uno de ellos se convertía en "bastón" de otras personas que estaban sufriendo de forma especial por la pandemia.

Tener la oportunidad de que sentirse útiles haciendo algo tan sencillo y a la par, tan significativo como escribir una carta de apoyo, ha sido muy enriquecedor en ambos sentidos (para el que la escribe y para el que la recibe) y más en un momento en el que las muestras de afecto no están permitidas: el contacto físico, los abrazos y los besos, la cercanía al hablar, el roce... las mascarillas, las medidas de seguridad y la distancia social se presentan como una barrera infranqueable para el encuentro interpersonal y sin embargo, a través de la palabra se ha generado un vínculo entre emisor y receptor más allá de todos esos impedimentos.

Adaptación de una materia universitaria al aprendizaje en línea en tiempos de COVID19: una oportunidad de mejora. De la Calle, Miró, de Dios y de la Rosa. 
Los medios de comunicación han enfatizado estos meses el dolor del ser humano al enfrentarse en soledad, a la enfermedad, al miedo, al confinamiento. Los alumnos de la Facultad de Comunicación han experimentado la importancia de sostener un vínculo con otro ser humano, han aprendido a comunicarse utilizando el soporte que fuera necesario para hacer llegar al otro una muestra del afecto humano cuando el abrazo no es posible. Saint-Exupéry en su libro Tierra de los hombres escrito tras cinco días de coma después de sufrir un accidente de avión, refleja la importancia comunicativa de un gesto. Relata la experiencia de un beduino que en mitad del desierto ofrece su agua a dos accidentados que temen por su vida. No se conocían, pero en el rostro de aquel hombre del desierto, el protagonista reconoce la bondad de toda la raza humana (Saint-Exupéry, 2016). Algo parecido han vivido nuestros estudiantes, como ellos mismos han comentado.

Los futuros profesionales del periodismo han comprendido la importancia de la comunicación interpersonal, la han descubierto in situ y en una situación excepcional. Han sido capaces de utilizar los recursos disponibles, desde los más sofisticados a los más tradicionales, y convertirlos en herramientas para lo verdaderamente relevante: acompañar, llevar un mensaje de aliento, de compañía y de esperanza a los que están pasando por una situación de mayor vulnerabilidad.

\section{Objetivos}

La pretensión de nuestro estudio es analizar la experiencia vivida por los estudiantes de periodismo y su respuesta de aprendizaje, como consecuencia de la necesaria adaptación de la asignatura de Responsabilidad Social ante la nueva situación académica provocada por la pandemia COVID-19.

En la asignatura de Responsabilidad Social se trabaja en la adquisición y desarrollo de competencias a través de la reflexión profunda sobre los pilares de la Ética y Deontología profesional, para lograr la interiorización del compromiso social y el del valor de darse a los demás y ejercer la labor profesional desde y para el bien común (De la Calle, 2009).

Esta materia pretende potenciar las habilidades propuestas por la Organización de las Naciones Unidas para la Educación, la Ciencia y la Cultura (UNESCO), en su informe de 1994: "La educación encierra un tesoro", en el que se defienden cuatro pilares básicos para formar: aprender a ser, aprender a conocer, aprender hacer y aprender a convivir (UNESCO, 1994).

El objetivo del presente estudio es identificar la manera en que los estudiantes universitarios de periodismo integran los conceptos teóricos relacionados con la responsabilidad social y comprobar el grado de desarrollo de las competencias comunicativas adecuadas, para transmitir su compromiso social, sobre todo en contextos de incertidumbre y en relación con colectivos vulnerables como el de las personas mayores, afectados de manera especial por el coronavirus.

Adaptación de una materia universitaria al aprendizaje en línea en tiempos de COVID19: una oportunidad de mejora. De la Calle, Miró, de Dios y de la Rosa. 


\section{Metodología}

Para llevar a cabo la investigación se ha empleado un método de análisis siguiendo un modelo de investigación cualitativa (López-Aranguren, 2015) apropiado para adecuarse al objeto de estudio: la experiencia de comunicación en el entorno de las relaciones sociales entre los alumnos de periodismo y las personas implicadas en sus prácticas de acción social. Dentro de ese modelo de investigación cualitativa se ha introducido un enfoque mixto al utilizar una cuantificación de la aparición de distintos términos que describen la experiencia de los alumnos a través del material obtenido en documentos escritos por los alumnos de la Facultad de Comunicación. En ellos se muestra de qué manera se han convertido en agentes de cambio social en medio de este estado de alarma y han llevado a la práctica su compromiso personal con las personas más vulnerables.

Para el análisis se ha usado un software específico para el análisis de datos, Atlas/ti en su versión 8. (Friese, 2019) Su adecuación óptima al tratamiento y análisis de datos a partir de la revisión de documentos escritos producidos por los alumnos ha servido para facilitar el análisis y la descripción de los resultados. Estos son presentados de forma clara a través de una herramienta de gran funcionalidad en el diseño de gráficos y nubes de palabras visualmente atractivos. El enfoque mixto de investigación cuenta con datos cuantificados dentro de un modelo cualitativo de estudio que utiliza el análisis de documentos y la observación directa como técnicas fundamentales.

El Atlas-ti es un programa computacional utilizado principalmente, pero no exclusivamente, en investigación cualitativa o análisis de datos cualitativos (Enver, 2017). Esta herramienta es muy reconocida por su capacidad de examen de los datos cualitativos en ciencias sociales, permitiendo manejar un gran número de palabras y documentos (San Martín, 2014). Con el soporte tecnológico del software ATLAS.ti, se realizó el análisis de contenido de los textos. Se logró construir evidencia empírica a partir de la base de datos generada y se identificaron conceptos, categorías y variables de análisis sobre el objeto de estudio.

Dentro del ámbito universitario hay otras investigaciones que ya han usado este software, optando por la red semántica (Cabero et al. 2020), lo que hace entender que es un método de investigación cada vez más arraigado en las universidades (López Meneses \& Jaén, 2012). El nuevo escenario docente, intervenido cada vez más por la transformación tecnológica, propicia el diseño de entornos de aprendizaje diferentes y ponen a nuestra disposición numerosas e interesantes posibilidades para enriquecer los procesos de innovación y de investigación (Domínguez et al. 2010; Morín \& Delgado, 2017; Álvarez Herrero, 2020).

Para el proceso de análisis se ha decidido estudiar todas las comunicaciones que los alumnos de periodismo han enviado a las personas mayores confinadas en las habitaciones de sus residencias. Las comunicaciones se establecieron con las instituciones sociales y residencias de la tercera edad con las que la UFV tiene un convenio de colaboración.

Adaptación de una materia universitaria al aprendizaje en línea en tiempos de COVID19: una oportunidad de mejora. De la Calle, Miró, de Dios y de la Rosa. 
Se han analizado con la herramienta o "Nube de palabras" que se usa para localizar las palabras claves dentro de los documentos trabajados (Vera et al. 2020). Si bien las nubes de palabras no son un método de análisis en sí mismo, sí son un método de presentación de resultados de fácil percepción. Esta forma de presentación de resultado permite entender en qué medida y forma el alumnado ha interiorizado los contenidos teóricos y ha sido capaz o no de plasmarlo en palabras.

Para procesar toda la información se ha creado un único documento donde se han recopilado todas las comunicaciones de los alumnos con las personas mayores y así poder manejar la documentación de forma más ágil.

Para el tratamiento de toda la información se ha creado un único documento donde se han recopilado todas las comunicaciones de los alumnos con las personas mayores y así poder manejar la documentación de forma más ágil. El procedimiento de análisis ha sido el realizado por varias personas para conseguir una mayor fiabilidad y valide de los análisis efectuados.

Se ha partido de la población de 42 alumnos de $2^{\circ}$ curso del grado de periodismo de la UFV (que se integra dentro de la Facultad de Comunicación en la que conviven distintos grados: Periodismo, Comunicación Audiovisual, Publicidad, Diseño...) y analizado una muestra de 42 documentos de 42 alumnos y 7322 palabras. Para obtener los resultados finales se estableció un umbral de repetición de 3 a 69, descartando las palabras que se repetían menos de 3 veces, por resultar irrelevantes para el estudio.

Además de la técnica "Nube de palabras" se han elaborado dos tablas, una con mayor índice de repetición y otra con menor, para su posterior análisis y relación con la parte teórica de la asignatura.

\section{Discusión y resultados}

Arenas aconseja que para medir el impacto de proyectos vinculados a áreas de responsabilidad social, se sigan las técnicas de codificación primaria y codificación secundaria para la recogida de datos; se proceda a la interpretación de los mismos y, por último, se proceda a la construcción de teorías según los resultados obtenidos (2012). Sin embargo, en los últimos años, el uso del software Atlas/ti para este tipo de investigaciones es cada vez más utilizado por parte de las universidades y facilita las labores interpretativas de los resultados (López Meneses \& Jaén, 2012; Álvarez Herrero, 2020).

En el programa informático utilizado, los resultados se presentan de tal manera que las palabras repetidas aparecen en un tamaño mayor y según se van nombrando menos se va reduciendo su tamaño. Se han quitado las palabras insignificantes para esta investigación como, preposiciones, artículos, adverbios... al igual que se ha seleccionado la no diferenciación de la mayúscula para que reconozca la palabra como un mismo conjunto.

Adaptación de una materia universitaria al aprendizaje en línea en tiempos de COVID19: una oportunidad de mejora. De la Calle, Miró, de Dios y de la Rosa. 


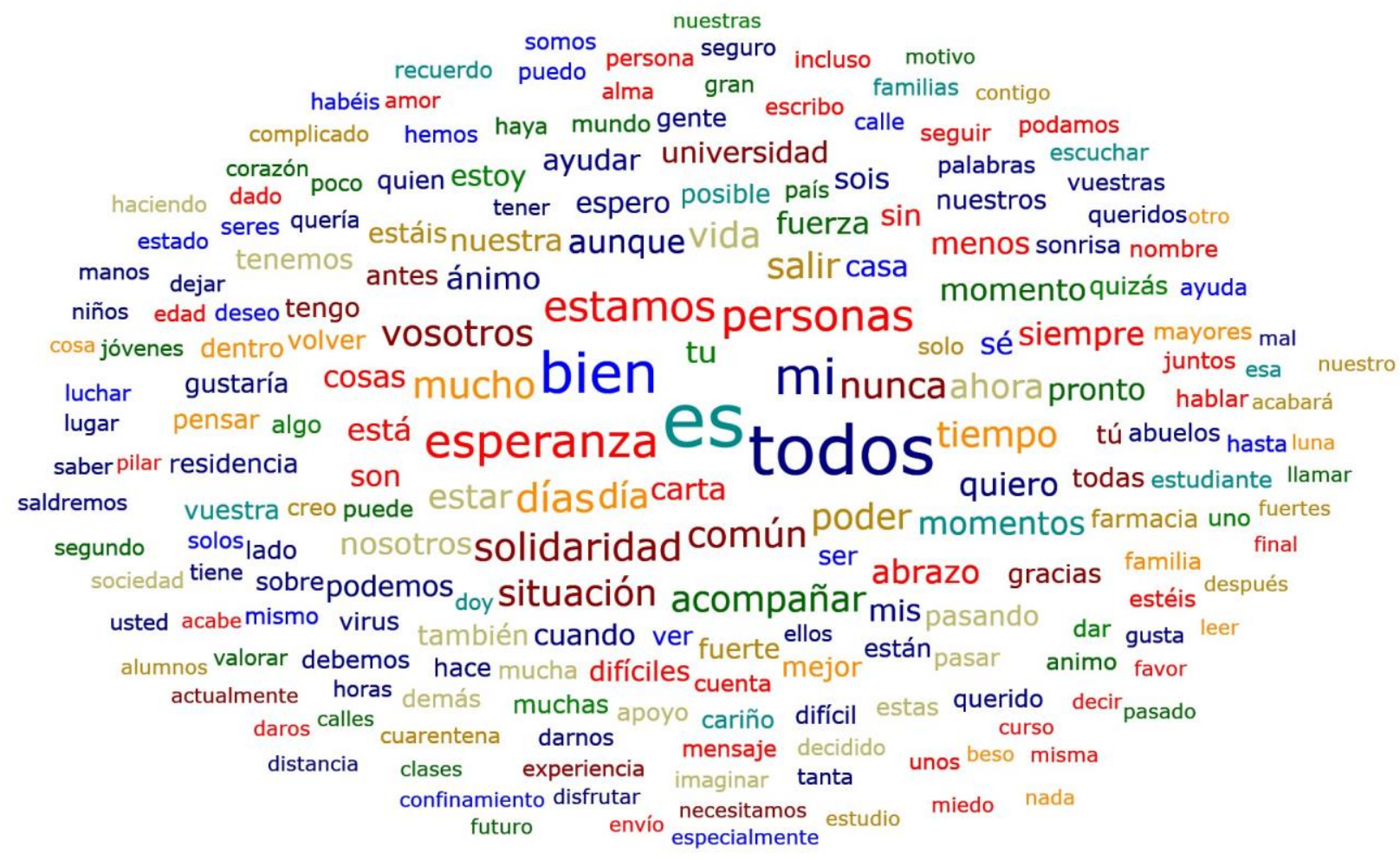

Figura 1. Resultado análisis

Fuente. Elaboración propia. Atlas.ti 8

Se han creado dos grupos de palabras para su análisis, las nueve palabras que están entre el umbral 21 y 60, es decir, con mayor índice de repetición.

Tabla 1. Términos más utilizados

\begin{tabular}{|c|c|}
\hline Palabra & Número \\
\hline Todos & 60 \\
\hline Bien & 46 \\
\hline Esperanza & 34 \\
\hline Persona & 30 \\
\hline Solidaridad & 25 \\
\hline Común & 22 \\
\hline Acompañar & 21 \\
\hline Tiempo & 21 \\
\hline Vida & 21 \\
\hline
\end{tabular}

Adaptación de una materia universitaria al aprendizaje en línea en tiempos de COVID19: una oportunidad de mejora. De la Calle, Miró, de Dios y de la Rosa. 
En este caso se observa como las palabras más repetidas son: Todos, Bien, Esperanza, Persona, Solidaridad, Común, Acompañar, Tiempo, Vida. Parece existir una relación directa con los contenidos tratados en la asignatura de Responsabilidad Social, que se detallan en el marco teórico y que se aterrizan en el conocimiento de las personas, la orientación al bien común y la esperanza de construir un mundo más solidario y justo para todos, términos que son repetidos de forma sistemática por el alumnado, lo que nos hace concluir que han asimilado la parte teórica de la materia. repetición.

Y otro grupo de nueve palabras entre el umbral 8 y 16, con menor índice de

Tabla 2. Términos con frecuencia media

\begin{tabular}{|c|c|}
\hline Palabra & Número \\
\hline Ánimo & 16 \\
\hline Universidad & 14 \\
\hline Fuerza & 15 \\
\hline Gracias & 11 \\
\hline Ayuda & 13 \\
\hline Podemos & 13 \\
\hline Mejor & 12 \\
\hline Corazón & 8 \\
\hline Cariño & 8 \\
\hline
\end{tabular}

En este caso las palabras que más se repiten son: Ánimo, Universidad, Fuerza, Gracias, Ayuda, Podemos, Mejor, Corazón, Cariño. Cariño. Otra de las apuestas de la materia, es la necesidad de inculcar en los universitarios, la importancia de su rol para mejorar el entorno y como con la ayuda de todos se puede conseguir un mundo mejor. Se observa en esta ocasión que las palabras menos citas por los estudiantes, también refleja el compromiso social del alumnado, que después de cursar la asignatura, con las metodologías expuestas anteriormente asume como propios los valores éticos aprendidos, adquiriendo habilidades y actitudes sociales (Rodríguez-Izquierdo, 2020).

Es necesario añadir algunas frases textuales de los documentos analizados ya que se creen evidencian algunos de los objetivos propuesto por la materia y explicados en el marco teórico "Os animo a mantener la juventud en los ojos, a burlarse del reloj que nunca para”, “... nunca perdáis la esperanza. Y no una esperanza fugaz, sino una esperanza vital para salir delante de este y de todos los problemas que puedan surgir en el futuro", "Volverá a salir el sol y lo volveremos a ver", "Cuando acabe todo esto seguiré yendo a veros como he estado haciendo todo este año", "Os echo mucho de menos", "Vosotros que sois flecha que nos guía en el camino cuando estamos perdidos", "Echamos de menos esas tardes en las que un solo café con vosotros se transformaba en un día de aventuras e historias, un día de aprender, un día de querer, pero, sobre todo, un día que disfrutar".

Adaptación de una materia universitaria al aprendizaje en línea en tiempos de COVID19: una oportunidad de mejora. De la Calle, Miró, de Dios y de la Rosa. 
Cabe añadir a estos resultados la observación de los alumnos que participaron en esta experiencia, que semanas más tarde, cuando la situación sanitaria lo permitía, alentados por la universidad, acudieron físicamente como voluntarios a repartir comidas, descargar alimentos, preparar menús...

El alumnado y profesorado de la asignatura aportó soluciones a la crisis social y sanitaria desde el área de conocimiento de cada grado; así por ejemplo: el equipo docente de la Escuela Politécnica Superior creó una plataforma de gestión integrada de poblaciones de riesgo; con el fin de desarrollar protocolos tempranos, optimizando e interconectando los recursos existentes en base a las necesidades de la ciudadanía, contribuyeron así a la no saturación de los centros sanitarios. También diseñaron un controlador inteligente programable por patología de paciente, aplicado a un kit básico y económico de respiración de asistencia primaria y de bajo coste, con el fin de poder ofrecer respiradores a los pacientes que los necesitasen.

Otro equipo docente, decidió unirse para crear el equipo Anticovid e imprimir viseras para proteger a los que trabajan contra el virus en primera línea. Esta tarea fue posible a la suma de diferentes empresas privadas y públicas que no dudaron en apoyar la creación de estos materiales, facilitando que profesores y alumnos con impresoras 3D fabricaran en un momento de escasez materiales necesarios.

Los estudiantes integraron la responsabilidad social aprendida y, en un momento crítico, dieron un paso al frente tal y como les propone con la asignatura de Responsabilidad Social. Todo apunta que el cursar la materia les ayudó a entender la situación excepcional que vive el país y a asumir su compromiso como ciudadanos con el resto de la sociedad. Los universitarios españoles demandan una mayor formación para conseguir un mundo más justo y sostenible, invitando al profesorado a transformar la sociedad desde las aulas, como puede verse en el estudio (Valderrama-Hernández et al. 2020).

Si bien estos resultados no se entienden sin el compromiso y actitud de la comunidad docente de la materia, conocedores de diferentes estudios que demuestran la importancia del apoyo emocional y empatía al alumno y su efecto en el clima del aula generando mejores resultados tal y como se demuestra en el estudio (Alonso-Tapia \& Nieto, 2019). En esta línea parece oportuno citar el estudio sobre la motivación de los universitarios y el éxito académico (Gutiérrez \& Tomás, 2018), que parecen estar estrechamente ligados, si se entiende por éxito los resultados presentados, se puede concluir que la motivación extra de los docentes tuvo un efecto positivo en el grupo.

Se ha dicho que una de las competencias que trabaja la materia es la de aprender a aprender, ofreciendo a los alumnos la oportunidad de comprender el mundo desde la práctica, hay estudios que trabajan en la evaluación de dicha competencia, por lo que se propone para futuras investigaciones usar el modelo propuesto (Gargallo et al. 2020) y analizar los resultados. También puede ser interesante, para conocer el aprendizaje del

Adaptación de una materia universitaria al aprendizaje en línea en tiempos de COVID19: una oportunidad de mejora. De la Calle, Miró, de Dios y de la Rosa. 
alumnado, ofrecer a los alumnos la herramienta de autoevaluación de aprendizaje servicio universitario (López de Arana et al. 2020).

Será interesante comparar, en un futuro próximo, los resultados de este estudio con otras propuestas, como la que se hace a los estudiantes de periodismo respecto a la redacción de trabajos a través de una aplicación InContext (Lerma-Noriega et al. 2020). Y abrir nuevas líneas de investigación inversa: un trabajo de campo en el que sean las personas mayores quienes escriban a los estudiantes, lo que permitirá comparar las comunicaciones de ambos colectivos respecto al impacto de la pandemia.

\section{Conclusiones}

El tiempo de confinamiento por la COVID-19 ha supuesto una oportunidad excepcional para asumir en primera persona los valores que la asignatura de Responsabilidad Social de la UFV propone a los alumnos que se preparan para ser profesionales de la comunicación. La situación actual provocada por la pandemia ha suscitado, aunque resulte paradójico, una renovación para la asignatura de Responsabilidad Social y ha enriquecido la experiencia de implicación del universitario en la búsqueda de soluciones colaborativas y de ayuda, reforzando en ellos el compromiso social. El objeto de esta investigación era hacer un análisis de la experiencia vivida por los estudiantes de periodismo, evaluar los resultados de aprendizaje ante esta adaptación y comprobar si su efecto ha incidido positivamente sobre la formación de los valores personales relacionados con la solidaridad, el bien común y la deontología profesional.

Partíamos del relevante papel que los medios de comunicación están jugando en la interpretación de los hechos que van aconteciendo desde el momento en que surgió la pandemia. Hemos pasado de contemplarla como una gripe más a convertirla en cifras y datos estadísticos, olvidando las diversas repercusiones que ha tenido y sigue teniendo sobre la vida de las personas.

La COVID-19 ha sido un revulsivo en nuestros alumnos que al verse sometidos a una situación tan extraordinaria por su dureza, han podido ponerse al servicio de los más vulnerables, comunicándoles su cercanía a través de sus mensajes. Les han querido transmitir una esperanza: "no eres un número pese a que parece que algunos medios te traten así, sigues siendo tú y reconozco en ti tu dignidad personal". Y es ahí cuando unas actividades sociales tan diferentes, en comparación con otros cursos académicos anteriores, les han llevado a plantearse su vocación desde una perspectiva deontológica mucho más auténtica, por ser fruto de una experiencia vivida con especial intensidad.

Nuestros alumnos, futuros periodistas, a través de sus cartas, encuentros en la red, infografías, plataformas digitales... han querido personalizar los encuentros en la distancia con las personas que más estaban sufriendo en esta pandemia. Han entendido que, ante la frialdad de unos datos y estadísticas, la palabra adquiere unas propiedades, en cierta manera, sanadoras. Los univeristarios han sido conscientes de que se estaban dirigiendo a una persona con un nombre propio, con una historia personal única e irrepetible, con una familia, con una circunstancia particular y que ahora se encontraba

Adaptación de una materia universitaria al aprendizaje en línea en tiempos de COVID19: una oportunidad de mejora. De la Calle, Miró, de Dios y de la Rosa. 
aislada, confinada, rodeada de silencio y soledad.

Todo se orienta para que el futuro profesional del periodista comprenda la responsabilidad que asume con la sociedad en la búsqueda de la verdad con la noticia que comunica. En estos meses los alumnos han experimentado los silencios informativos, la falta de claridad, los datos contradictorios, los bulos... y, por otro lado, han comprobado el bien que proporciona un mensaje honesto cuando al redactarlo se tiene en cuenta verdaderamente al receptor como lo que es: nada más y nada menos que una persona, igual que el emisor, con los mismos miedos, ilusiones, sueños y pesadillas. Surge entonces un vínculo comunicativo capaz de crear una situación de auténtico encuentro y de cercanía, aunque se desconozca el nombre del que lo recibe, porque por encima de todo, se reconoce a cada persona como un ser humano necesitado de tu ayuda.

Nuestros estudiantes de periodismo han aprendido a valorar la necesidad de ser personas íntegras en el ejercicio de su profesión y de adoptar un sentido de responsabilidad social y de compromiso ético. El haber sufrido una situación de pandemia les ha llevado a la primera línea de acción antes de lo previsto. Como hemos visto en el análisis de datos anterior, el resultado para su formación ha sido verdaderamente fecundo.

En cuanto a la prospectiva, cabe señalar que hay estudios (Moller-Recondo \& D’Amato, 2020) que relacionan el talento universitario con el compromiso social, su autonomía a la hora de liderar su proceso de formación en primera persona y la formación en Antropología, tal y como propone la asignatura de Responsabilidad Social. Será relevante para próximos estudios hacer una comparativa con la propuesta hecha en el estudio de Johnson et al. (2020), que ha usado la misma metodología, pero con una finalidad distinta, pues su objetivo era conocer la percepción por parte de los ciudadanos de cómo vivían la pandemia.

Terminamos nuestro trabajo afirmando que cualquier situación de emergencia exige que cada ciudadano ponga en juego todos sus recursos para sacar lo mejor de sí mismo y ponerlo al servicio de los demás. La situación actual provocada por la pandemia ha supuesto, sin embargo y aunque resulte paradójico, una oportunidad para replantear la asignatura de Responsabilidad Social y ha enriquecido la experiencia de implicación del universitario en la búsqueda de soluciones colaborativas y de ayuda, reforzando en ellos el compromiso social. Nos proponíamos como objetivo de esta investigación hacer un análisis de la experiencia vivida por los estudiantes de periodismo y su respuesta de aprendizaje, como consecuencia de la necesaria adaptación de la asignatura de Responsabilidad Social ante la nueva situación académica provocada por la pandemia COVID-19.

De esta experiencia, podemos concluir que la universidad está llamada a salir al encuentro de aquellos sucesos que acontecen e impactan de forma inesperada y caótica en la sociedad, siempre desde la búsqueda honesta de la verdad a la hora de enseñar, investigar y divulgar, con ética y responsabilidad.

De la misma manera, los medios de comunicación tradicionales y las nuevas formas tecnológicas de información han de respetar un código deontológico que salvaguarde la veracidad de las noticias que difunden. Solo en la medida en que los

Adaptación de una materia universitaria al aprendizaje en línea en tiempos de COVID19: una oportunidad de mejora. De la Calle, Miró, de Dios y de la Rosa. 
periodistas e informadores sean conscientes de su papel protagonista y de su responsabilidad social, podrán contribuir con su ejercicio profesional a la búsqueda de soluciones eficaces para el bien común; especialmente en crisis como la que estamos viviendo en este momento por la pandemia del COVID 19.

Y es en la Universidad, como institución que busca la verdad y la sabiduría, donde han de ser formados esos universitarios socialmente responsables, que llevarán a cabo la valiosa labor de informar con rigor y veracidad el día de mañana desde los medios de comunicación. Solo así la deontología profesional dejará de ser una asignatura pendiente.

Presentación del manuscrito: 10 de octubre de 2020

Fecha de aprobación: 13 de noviembre de 2020

Fecha de publicación: 8 de enero de 2021

De la Calle, C., Miró, S., de Dios, T., y de la Rosa, D. (2021). Adaptación de una materia universitaria al aprendizaje en línea en tiempos de COVID-19: una oportunidad de mejora. RED. Revista Educación a Distancia, 21(65). http://dx.doi.org/10.6018/red.449841

\section{Financiación}

Investigación realizada por el GEI RSEU (Grupo estable de investigación sobre Responsabilidad Social del Estudiante Universitario) y financiada por el Instituto de Investigaciones Económicas y Sociales. Mayo de 2020 a julio de 2020.

\section{Referencias}

Adelson, L. (2020). La epidemia de desinformación por el coronavirus pide ciencia, solidaridad e información contrastada. Noticias ONU. Recuperado de: https://news.un.org/es/story/2020/04/1472822

Agejas, J.A. \& De Dios, T. (2016). El discernimiento prudencial en orden al ejercicio de la responsabilidad social del informador. En: R. Cetina, L. Corredoira \& Alfonso F. Gutiérrez (Eds.), Informar ¿derecho o deber?, 326-345. Madrid: Universidad Complutense de Madrid.

Alonso-Tapia, J. \& Nieto, C. (2019). Clima emocional de clase: naturaleza, medida, efectos e implicaciones para la educación. Revista Psicodidáctica. 24(2), 79-87. https://10.1016/j.psicod.2018.08.002

Álvarez, J.J. (2007). La verdad y la objetividad como premisas del actuar responsable de los periodistas. En: P. Giménez-Armentia, C. De la Calle \& L. Climent (Eds. y cood.), Persona y sociedad. Las dos caras del compromiso, 159-165. Madrid: Universidad Francisco de Vitoria.

Adaptación de una materia universitaria al aprendizaje en línea en tiempos de COVID19: una oportunidad de mejora. De la Calle, Miró, de Dios y de la Rosa. 
Álvarez Herrero, J. F. (2020). Tendencias en la tecnología educativa de nuestros días. Edetania. Estudios y Propuestas Socioeducativas, (56), 3-60. https://doi.org/10.46583/edetania_2019.56.506

Ballesteros, J. (2020). El papel de los medios: la comunicación oficial y no oficial en la crisis del coronavirus. La Huella Digital, 1-5. Madrid: Fundación Pablo VI. Recuperado de: https://www.fpablovi.org/images/actividades/huella/2020JuanBenavides.pdf

Bodenhausen, G.V. \& Peery, D. (2009). Social Categorization and StereotypingIn vivo: The VUCA Challenge. Social and Personality Psychology Compass, 3(2), 133151. https://doi.org/10.1111/j.1751-9004.2009.00167.x

Cabero, J., Vázquez-Cano, E., López Meneses, E. \& Jaén, A. (2020). Posibilidades formativas de la tecnología aumentada. Un estudio diacrónico en escenarios universitarios. Revista Complutense de Educación, 31(2), 143-154. Madrid: Universidad Complutense. https://doi.org/10.5209/rced.61934

Catalán-Matamoros, D. (2020). La comunicación sobre la pandemia del COVID-19 en la era digital: manipulación informativa, fake news y redes sociales. RECS, Revista Española de Comunicación en Salud, suplemento 1, 1-4. Madrid: Universidad Carlos III. https://doi.org/10.20318/recs.2020.5531

Cardoza, G. (2020). Los retos de la información y la comunicación en la era de la pandemia: el enfoque de design thinking. Forbes Centroamérica. Recuperado de: https://forbescentroamerica.com/2020/06/23/los-retos-de-la-informacion-y-lacomunicacion-en-la-era-de-la-pandemia-el-enfoque-de-design-thinking/

Centro de documentación europea. Universidad Francisco de Vitoria (2020). Recuperado en 2020 de https://centro-documentacion-europea-ufv.eu/alfabetizacioninformacional-union-europea/

Comisión Económica para Amérca Latina y el Caribe (CEPAL-UNESCO) (2020). La educación en tiempos de la pandemia de COVID-19. Informe COVID-19. Recuperado de: https://www.cepal.org/es/publicaciones/45904-la-educaciontiempos-la-pandemia-covid-19

Cordón, O., Alcalá, Á., Arenas, M., Camarillo, J., García, D. M., Gumbau, J. P., Martín, J. M., Martínez, R., Puig, M., Sampalo, F. \& Vendrell, E. (2020). Informe sobre Procedimientos de Evaluación no Presencial. Estudio del Impacto de su Implantación en las Universidades Españolas y Recomendaciones Versión 1.0. Madrid: CRUE Universidades Españolas. Recuperado de: https://internacional.unizar.es/sites/internacional.unizar.es/files/archivos/pdf/cov id/20200416_informe_procedimiento_evaluacion_online.pdf

Comisión Europea (2018). La lucha contra la desinformación en línea: un enfoque europeo. Bruselas. Recuperado de: https://eur-lex.europa.eu/legalcontent/ES/TXT/PDF/?uri=CELEX:52018DC0236\&from=PL

Adaptación de una materia universitaria al aprendizaje en línea en tiempos de COVID19: una oportunidad de mejora. De la Calle, Miró, de Dios y de la Rosa. 
De Dios, T. (2018). Responsabilidad de la persona y sostenibilidad de las organizaciones. Madrid: Universidad Francisco de Vitoria.

De la Calle, C. (2009). La formación de la responsabilidad social del universitario: un estudio empírico. Madrid: Universidad Complutense de Madrid.

De la Calle, C. \& Giménez-Armentia, P. (2011). Aproximación al concepto de Responsabilidad Social del Universitario. Revista Comunicación y hombre (7), 236-247.

Recuperado de: http://www.comunicacionyhombre.com/pdfs/07_i_delacalleyjimenez.pdf

Domínguez, G., López Meneses, E., Amador, L. \& Esteban, M. (Coords.). (2010). Experiencias universitarias innovadoras en educación social. Sevilla: Mergablum.

Du Preez, P. (2020). Noticias falsas y desinformación, otra pandemia del coronavirus. Noticias ONU. Recuperado de: https://news.un.org/es/story/2020/04/1472922

Friese, S. (2019). Qualitative Data Analysis with ATLAS.ti. California: Editorial Sage.

García Ramos, J.M., De La Calle, C., Valbuena, C. \& De Dios, T. (2016). La formación en Responsabilidad social y su impacto en diversas carreras universitarias. Revista de investigación educative, 34(2), 435-453. http://dx.doi.org/10.6018/rie.34.2.244271

García-Peñalvo, F.J., Corell, A., Abella-García, V. \& Grande, M. (2020). La educación online en la educación superior en tiempos de la COVID-19. Education in the Knowledge Society 21. https://doi.org/10.14201/eks.23013

Gargallo, B., Pérez-Pérez, C., García-García, F.J., Giménez, J.A. \& Portillo, N. (2020). The skill of learning to learn at university. Proposal for a theoretical model. Educación XX1, 23(1), 19-44. http://dx.doi.org/10.5944/educXX1.23367

Gutiérrez, M. \& Tomás, J.M. (2018). Clima motivacional en clase, motivación y éxito académico en estudiantes universitarios. Revista Psicodidáctica, 23(2), 94-101. http://dx.doi.org/10.1016/j.psicod.2018.02.001

Hernández de Hahn, L. (2014). Socially Responsible Citizens: Promoting Gifts and Talents That Support Social and Humanitarian Advancement. International Studies in Sociology of Education, 24(4), 415-434. http://dx.doi.org/10.1080/09620214.2014.979595

Hodges, C., Moore, S., Lockee, B., Trust, T. \& Bond, A. (2020). The difference between emergency remote teaching and online learning. Educause Review. Recuperrado de: https://bit.ly/3b0Nzx7

Adaptación de una materia universitaria al aprendizaje en línea en tiempos de COVID19: una oportunidad de mejora. De la Calle, Miró, de Dios y de la Rosa. 
Johnson, M. C., Saletti-Cuesta, L. \& Tumas, N. (2020). Emociones, preocupaciones y reflexiones frente a la pandemia del COVID-19 en Argentina. Ciência \& Saúde Coletiva, 25(1), 2447-2456. Epub June 05, 2020. https://doi.org/10.1590/141381232020256.1 .10472020

Landøy, A., Popa, D. \& Repanovici, A. (2020). Collaboration in Designing a Pedagogical Approach in Information Literacy. Texas: Springer open.

Larrán, M. (2014). Análisis del nivel de implantación de políticas de Responsabilidad Social en universidades españolas. Conferencia de Consejos Sociales de las Universidades Públicas Españolas. Las Palmas de Gran Canarias: Colección Experiencias y Resultados.

Lerma-Noriega, C.A., Flores-Palacios, M.L. \& Rebolledo-Méndez, G. (2020). InContext: Una aplicación móvil para mejorar las estrategias de aprendizaje en la universidad. Comunicar, 28(64), 109-118. https://doi.org/10.3916/C64-202010

López de Arana, E., Aramburuzabala Higuera, P. \& Opazo Carvajal, H. (2020). Design and validation of a questionnaire for self-assessment of university service-learning experiences. Educación XX1, 319-347. https://doi.org/10.5944/educXX1.23834

López Meneses, E. \& Jaén, A. (2012). Experiencia de innovación educativa con nubes. Revista Educativa Hekademos, 11(V), 59-66. Recuperado de: http://www.hekademos.com/hekademos/media/articulos/11/06.pdf

López Meneses, E., Llorent, V. \& Fernández, E. (2013). Experiencia universitaria sobre las funciones del educador/a social con tecnologías 2.0. EDUTEC. Revista Electrónica de Tecnología Educativa, 43, 1-17. Recuperado de: file://C:/Users/Usuario/Downloads/333-Texto\%20del\%20art\%C3\%ADculo875-1-10-20150311\%20(1).pdf

López Quintás, A. (2003). Inteligencia creativa: el descubrimiento personal de los valores. Madrid: B.A.C.

López-Aranguren, E. (2015). El análisis de contenido tradicional. En: M. García Ferrando, F.R. Alvira, L. E. Alonso \& R. Modesto (Coords.), El análisis de la realidad social: métodos y técnicas de investigación. Madrid: Alianza.

Marcos, A. (2020). Con COVID y sin COVID: La vulnerabilidad humana. Cuadernos de Bioética, 31 (102), 139-149. https://doi.org/10.30444/CB.58

Moller-Recondo, C. \& D’Amato, J.P. (2020). Nuevos genios emprendedores: Itinerario y trayectorias de excelencia educativa universitaria. Comunicar, 28(64), 73-83. https://doi.org/10.3916/C64-2020-07

Adaptación de una materia universitaria al aprendizaje en línea en tiempos de COVID19: una oportunidad de mejora. De la Calle, Miró, de Dios y de la Rosa. 
Morelo, G, Durán, O. \& Abu, L. (2020). Enseñar periodismo en tiempos de coronavirus: un reto de dimensiones pandémicas. Red Ética. Recuperado de: https://fundaciongabo.org/es/etica-periodistica/entrevistas/ensenar-periodismoen-tiempos-de-coronavirus-un-reto-de-dimensiones

Morín, E. \& Delgado, C. J. (2017). Reinventar la educación. Abrir caminos a la metamorfosis de la humanidad. Cuba: Editorial UH.

Ortiz De Montellano, S. (2000). Evaluación de la responsabilidad social del egresado universitario. Madrid: Universidad Complutense de Madrid, Facultad de Educación.

Ortiz, T. \& Sanz, T. (2016). Visión pedagógica de la formación universitaria actual. Cuba: Editorial UH.

Osorio, M. (2003). Voluntariado social. España: Centro de Estudios Ramón Areces.

Reason, R. D., Ryder, A. J. \& Kee, C. (2013). Higher education's role in educating for personal and social responsibility: A review of existing Literature. New Directions for Higher Education, (164), 13-22. https://doi.org/10.1002/he.20072

Rodriguez-Izquierdo, R.M. (2020). Aprendizaje Servicio y compromiso académico en Educación Superior. Revista de Psicodidáctica. 25(1), 45-51. https://doi.org/10.1016/j.psicod.2019.09.001

Sáenz, C. (2012). Medición de los impactos de la difusión de la innovación de un proyecto de Responsabilidad Social. Recuperado de: https://www.tdx.cat/bitstream/handle/10803/83607/S\%C3\%81ENZ\%20ACOST A_Tesis\%20Doctoral_2012-04-09_FV.pdf?sequence=1\&isAllowed=y

Saint-Exupéry, A. (2016). Tierra de los hombres. Córdoba: Ed. Berenice.

San Martín, D. (2014). Teoría fundamentada y Atlas.ti: recursos metodológicos para la investigación educativa. REDIE, 16(1), 104-122. Recuperado de: http://www.scielo.org.mx/scielo.php?script=sci_arttext\&pid=S160740412014000100008

Terol-Bolinches, R. \& Alonso-López, N. (2020) La prensa española en la Era de la Posverdad: el compromiso de la verificación de datos para combatir las Fake News. Revista Prisma Social, 31, 304-327. Recuperado de: https://revistaprismasocial.es/article/view/3924/4549.

UNESCO. (1994). The four pillars of education, Education: The Treasure Within. El Correo de la UNESCO, 91-103. Recuperado de: https://www.gcedclearinghouse.org/sites/default/files/resources/\%5BENG\%5D \%20Learning_0.pdf

Adaptación de una materia universitaria al aprendizaje en línea en tiempos de COVID19: una oportunidad de mejora. De la Calle, Miró, de Dios y de la Rosa. 
Valderrama-Hernández, R., Alcántara, L., Sánchez-Carracedo, F., Caballero, D., Serrate, S., Gil-Doménech, D., Vidal-Raméntol, S. \& Miñano, R. (2020). Does the Spanish university system teach sustainability? Perception of the students of four universities. $\quad$ Educación $\quad X X 1, \quad 23(1), \quad$ 221-245. https://doi.org/10.5944/educXX1.23420

Vega, E. (2017). Análisis de contenido: Análisis estadístico de textos en Atlas.ti. Lima: Pontificia Universidad Católica del Perú. Recuperado de: https://www.researchgate.net/publication/327793117_Analisis_de_contenido_A nalisis_estadistico_de_textos_en_Atlasti

Vera, S., Villao, D.C. \& Granados, J.F. (2020). Competencias digitales en el uso de herramientas digitales para el aprendizaje de inglés. Leoncio Ingenio, 1(3). https://doi.org/10.18779/ingenio.v3i1.21

Adaptación de una materia universitaria al aprendizaje en línea en tiempos de COVID19: una oportunidad de mejora. De la Calle, Miró, de Dios y de la Rosa. 